\title{
Semi-continuous methane production from undiluted brown algae using a halophilic marine microbial community
}

Toyokazu Miura $^{\mathrm{a}, \mathrm{c}}$, Akihisa Kita ${ }^{\mathrm{a}, \mathrm{c}}$, Yoshiko Okamura, ${ }^{\mathrm{a}, \mathrm{c}}$, Tsunehiro Aki ${ }^{\mathrm{a}, \mathrm{c}}$, Yukihiko Matsumura $^{\mathrm{b}, \mathrm{c}}$, Takahisa Tajima ${ }^{\mathrm{a}, \mathrm{c}}$, Junichi Kato ${ }^{\mathrm{a}}$, Yutaka Nakashimada ${ }^{\mathrm{a}, \mathrm{c}, *}$

a Department of Molecular Biotechnology, Graduate School of Advanced Sciences of Matter, Hiroshima University, 1-3-1 Kagamiyama, Higashi-Hiroshima 739-8530, Japan

${ }^{\mathrm{b}}$ Division of Energy and Environmental Engineering, Institute of Engineering, Hiroshima University, 1-4-1 Kagamiyama, Higashi-Hiroshima 739-8527, Japan

${ }^{\mathrm{c}}$ CREST, JST

*Corresponding author at: Department of Molecular Biotechnology, Graduate School of Advanced Sciences of Matter, Hiroshima University, 1-3-1 Kagamiyama, Higashi-Hiroshima 739-8530, Japan. Tel./fax: +81 824244443.

E-mail address: nyutaka@ hiroshima-u.ac.jp (Y. Nakashimada) 


\begin{abstract}
Acclimated marine sediment-derived culture was used for semi-continuous methane production from materials equivalent to raw brown algae, without dilution of salinity and without nutrient supply, under 3 consecutive conditions of varying organic loading rates (OLRs) and hydraulic retention time (HRT). Methane production was stable at 2.0 g VS/kg/day (39-day HRT); however, it became unstable at $2.9 \mathrm{~g} \mathrm{VS} / \mathrm{kg} /$ day (28-day HRT) due to acetate and propionate accumulation. OLR subsequently decreased to $1.7 \mathrm{~g}$ VS/kg/day (46-day HRT), stabilizing methane production beyond steady state. Methane yield was above $300 \mathrm{~mL} / \mathrm{g}$ VS at all OLRs. These results indicated that the acclimated marine sediment culture was able to produce methane semi-continuously from raw brown algae without dilution and nutrient supply under steady state. Microbial community analysis suggested that hydrogenotrophic methanogens predominated among archaea during unstable methane production, implying a partial shift of the methanogenic pathway from acetoclastic methanogenesis to acetate oxidation.
\end{abstract}

Keywords: Semi-continuous methane production; Brown algae; Undiluted conditions; Microbial community 


\section{Introduction}

Marine macroalgae are receiving increasing attention as a feedstock for biomethane production because they do not compete with food for farm land, have a higher growth rate than terrestrial energy crops, and are less resistant to degradation than lignocellulosic feedstocks (Montingelli et al., 2015). Methane can be produced by either thermal or biological gasification of biomass (Chynoweth et al., 2001). Marine macroalgae are suitable for biological gasification by anaerobic microbes because marine macroalgae have a high water content of around 90\% (Roesijadi et al., 2010), which is not suitable for the thermal process requiring feedstocks with a low water content of less than $50 \%$ to achieve the high temperature needed for the process (Chynoweth et al., 2001). However, marine macroalgae contain high levels of salts (Roesijadi et al., 2010), which inhibit methane production (Feijoo et al., 1995; Liu and Boone, 1991; Miura et al., 2014; Mottet et al., 2014; Rinzema et al., 1988). Therefore, the use of diluted feedstocks or halophilic microbes is needed to allow methane production from these feedstocks.

Methane has been produced from marine macroalgae under semi-continuous conditions using diluted feedstocks (Briand and Morand, 1997; Hanssen et al., 1987; Hinks et al., 2013). In these conditions, the salinity of the feedstock decreases; however, the organic matter in the feedstocks concomitantly decreases because of increased water content, resulting in decreased organic loading rate (OLR) at the same hydraulic retention time (HRT). To obtain the same OLR, the HRT must be kept shorter under diluted than under undiluted conditions, which can result in excessively short HRT causing washout of microbes in traditional single-stage methane production. Therefore, 
methane should be produced under undiluted conditions to obtain a high OLR without excessively shortening the HRT.

Miura et al. (2015) reported the acclimation of a microbial community from marine sediments by fed-batch cultivation for high-rate, continuous methane production from undiluted raw brown algae. The acclimation of the sediment by fed-batch cultivation enabled obtaining an increased methane production rate compared to the initial marine sediment slurry despite the presence of excess salts in the raw brown algae in addition to the $3 \% \mathrm{NaCl}$ present in the initial slurry. However, it remains to be evaluated whether the acclimated microbial community is actually applicable to continuous methane production from raw brown algae.

The aim of this study was to evaluate the ability of the acclimated culture to produce methane continuously from brown algae under undiluted conditions. For this purpose, anaerobic digestion was conducted in a semi-continuous regime, using materials equivalent to undiluted raw brown algae, without nutrient supply. The OLR was varied during the production, which was continued beyond steady state.

\section{Methods}

\subsection{Experimental design}

Semi-continuous cultivation was carried out using acclimated marine sediment-derived culture as the starter culture. The starter culture was initiated in triplicate in 700-mL vials with intermittent replacement with fresh materials (dried brown algae and anaerobic water) with a total solid (TS) content of $10 \mathrm{wt} \%$, which is equivalent to that of raw brown algae, for semi-continuous methane production. The cultures removed from the triplicate cultures at replacement were combined to measure 
the volatile solid (VS) content. An aliquot was taken at the beginning of cultivation with fresh materials to measure the $\mathrm{pH}$ and salinity. Sampling was also conducted at the end of the cultivation to measure the volatile fatty acids (VFAs) and to analyze microbial community.

\subsection{Materials}

Dried and milled Saccharina japonica was used as substrate (Miura et al., 2015). The TS, VS, and chemical oxygen demand (COD) of the substrate were $94.2 \mathrm{wt} \%, 73.3 \mathrm{wt} \%$, and $1030 \mathrm{mg} / \mathrm{g}$ TS, respectively.

\subsection{Semi-continuous methane production using a fed-batch acclimated culture}

The starter culture for semi-continuous methane production was prepared in accordance with a previously described protocol for fed-batch acclimation (Miura et al., 2015). In brief, marine sediment and dried $S$. japonica were used as the microbial source and substrate, respectively. Microbes in the marine sediment were acclimated by fed-batch cultivation with repeated addition of dried brown algae to the sediments at 1 wt $\%$ of water amount. Thirteen cycles of cultivation were used; anaerobic water was added at the beginning of the $11^{\text {th }}$ round to decrease the salinity and to examine the effect of salinity on the rate of methane production. The cultivation was conducted in triplicate. The salinity at the beginning of $13^{\text {th }}$ round culture was equivalent to approximately $4 \% \mathrm{NaCl}$.

Batch cultivation was conducted before starting semi-continuous cultivation to examine the methane productivity. The substrate was added at $1.5 \mathrm{~g}$ TS to the starter culture (approximately $200 \mathrm{~g}$ ) in 700 -mL vials. Anaerobic water prepared under $\mathrm{N}_{2} / \mathrm{CO}_{2}$ 
(80:20) was added so that the materials added had a TS content of $10 \mathrm{wt} \%$, equivalent to the TS content of raw brown algae. Cultures were incubated statically at $37{ }^{\circ} \mathrm{C}$ in triplicate.

Semi-continuous cultivation was conducted under 3 consecutive conditions of varying OLR and HRT as follows: condition 1, OLR of $2.0 \pm 0.1 \mathrm{~g}$ VS substrate/kg culture/day and HRT of $39.2 \pm 1.9$ days; condition 2 , OLR of $2.9 \pm 0.2 \mathrm{~g} \mathrm{VS}$ substrate/kg culture/day and HRT of $27.8 \pm 1.6$ days; and condition 3, OLR of $1.7 \pm$ $0.02 \mathrm{~g}$ VS substrate/kg culture/day and HRT of $45.8 \pm 0.8$ days (Fig. 1a). Culture was removed semi-continuously using a tube (3-mm internal diameter) connected with a 20-mL syringe, and replaced with fresh materials (substrate and anaerobic water) with a substrate-TS content of $10 \mathrm{wt} \%$. The cultures were incubated statically at $37^{\circ} \mathrm{C}$ and conducted in triplicate.

The OLR was calculated from the VS of substrate added, the weight of the culture measured at the start of cultivation with fresh materials, and the period of cultivation. The OLR at each cultivation with fresh materials was the average of triplicate cultures. The total OLR during cultivation in each of the cultivation conditions was expressed as the average \pm standard deviation (SD). The HRT was calculated from the ratio of the weight of fresh materials to the weight of culture measured at the start of cultivation and the period of cultivation. The HRT at each cultivation with fresh materials was the average of triplicate cultures. The total HRT during cultivation in each of the cultivation conditions was expressed as the average \pm SD.

The methane yield in the semi-continuous process was calculated as the slope of the plot of the cumulative amount of methane produced against the cumulative amount of substrate added. 
For measurement of acetoclastic methanogenic activity, $9.4 \mathrm{~mL}$ of medium containing sodium acetate used as substrate (Miura et al., 2014) and $0.6 \mathrm{~mL}$ of culture from the first round of semi-continuous cultivation under condition 3 were mixed to make up $10 \mathrm{~mL}$ of culture containing $5 \mathrm{~g} / \mathrm{L}$ of sodium acetate. Triplicate cultures were incubated statically in a test tube at $37^{\circ} \mathrm{C}$. The activity was measured under different salinity levels of $0 \%, 1 \%, 2 \%, 3 \%$, and $4 \% \mathrm{NaCl}$. The ratio of substrate to inoculum was 5 to 1 on the basis of the VS. The acetoclastic methanogenic activity was calculated from the plot of acetate concentration against cultivation day by fitting a third-polynormal trendline. The maximum slope of the trendline was calculated as the activity.

\subsection{Chemical analyses}

The VS, COD, VFAs, methane, the rate of methane production, and salinity were analyzed as reported previously (Miura et al., 2015). Briefly, the VS content of the substrate and culture was measured after incineration at $600{ }^{\circ} \mathrm{C}$ of the TS, which was dried at $105{ }^{\circ} \mathrm{C}$. The VFAs were analyzed by high-performance liquid chromatography using crotonate as the internal standard. The methane content was measured using a gas chromatograph and the total gas volume was determined by substituting the gas with water in a cylinder. The rate of methane production was calculated using the modified Gompertz equation from plots of the methane yield. The salinity was calculated from the standard curve of conductivity and the concentration of $\mathrm{NaCl}$. The $\mathrm{pH}$ of the culture supernatant was measured by using a pH meter (LAQUAtwin B-712; Horiba, Kyoto, Japan). 


\subsection{Microbial community analysis}

Triplicate culture samples were taken from the starter culture and at the end of culture under conditions 1, 2, and 3 using an 18-gauge needle, and they were stored at $-20^{\circ} \mathrm{C}$. These samples (weighing about $250 \mathrm{mg}$ each) were subjected to genomic DNA extraction and subsequent 16S rRNA gene amplification as reported previously (Miura et al., 2015) except for the barcode sequences used in the amplification. The appearance of the samples was different depending on the contents of marine sediment. Although the starter culture consisted of marine sediment, the amount of sediment contained in these samples decreased with progressing conditions because the starter culture was replaced with fresh substrate during the various stages of the semi-continuous operation. However, this variation in the sediment contents probably did not affect subsequent genomic DNA extraction because the sediment amount in the samples used for extraction was within the linear region of the plot of extracted DNA amount versus the sediment amount. The barcode sequences used were as follows: 5'-TAGTGTAGAT-3' for bacteria and 5'-TGCTATTCAG-3' for archaea in the starter culture, 5'-TACAGATCGT-3' for bacteria and 5'-TGCTATTCAG-3' for archaea at the end of culture under condition 1, 5'-TACGCTGTCT-3' for bacteria and 5'-TGCTAGTCAG-3' for archaea at the end of culture under condition 2, 5'-TCGATCACGT-3' for bacteria and 5'-TGCTAGTCAG-3' for archaea at the end of culture under condition 3. Triplicate amplicons were combined, and a single sample per condition was subjected to microbial community analysis at the Hokkaido System Science (Hokkaido, Japan). This involved pyrosequencing and the Quantitative Insights into Microbial Ecology (QIIME) pipeline as reported previously (Miura et al., 2015). Amplicons obtained from the starter culture and the culture under condition 3 were simultaneously sequenced using the Roche GS 
FLX+ system. Amplicons obtained from the cultures under conditions 1 and 2 were sequenced in independent runs using the Roche GS Junior.

The relative abundance of taxa within each sample was calculated by dividing the number of reads for each taxon by the total number of reads per sample.

\section{Results and discussion}

\subsection{Semi-continuous methane production}

\subsubsection{Stability of semi-continuous methane production}

Semi-continuous cultivation was conducted using a fed-batch acclimated marine sediment starter culture and fresh materials (substrate and anaerobic water) equivalent to raw brown algae as the substrate under varying OLR and HRT (Fig. 1a). First, batch cultivation was conducted to examine the methane productivity (Fig. 1b). The same amount of substrate (1.5 g TS) as the amount used in the fed-batch acclimation was added to the starter culture. The methane yield from $1.5 \mathrm{~g}$ TS of substrate was $458 \pm 10$ $\mathrm{mL}$, which corresponds to $386 \pm 9 \mathrm{~mL} \mathrm{CH}_{4} / \mathrm{g}$ VS and $79.1 \pm 1.8 \% \mathrm{COD}$, after 6.9 days of cultivation. After 3 days of cultivation, the methane yield was $393 \pm 14 \mathrm{~mL}$, corresponding to $331 \pm 12 \mathrm{~mL} \mathrm{CH}_{4} / \mathrm{g}$ VS and $67.9 \pm 2.4 \% \mathrm{COD}$ of substrate. Because of the high performance of the methanogenic microorganisms in batch cultivation, semi-continuous cultivation was subsequently conducted.

Semi-continuous cultivation was started with intermittent replacement of part of the culture with fresh materials. Cultivation was started under condition 1 (OLR of $2.0 \mathrm{~g}$ VS substrate/kg culture/day and HRT of 39 days), where the amount of replaced materials (substrate and anaerobic water) contained $1.5 \mathrm{~g}$ TS of substrate and the cultivation period was approximately 2.9 days (Fig. 1a). VFA did not accumulate, and 
the methane production was generally stable although the production seemed to be unstable in the initial phase, between the first and eighth addition of fresh materials, because of a gas leak owing to a damaged butyl rubber tube (Fig. 1b). Therefore, the methane yield was calculated between the ninth and last addition of fresh materials, and was $358 \pm 1 \mathrm{~mL} \mathrm{CH} / \mathrm{g} \mathrm{VS}$.

To produce methane at a higher OLR, cultivation was continued under condition 2 (OLR of $2.9 \mathrm{~g} \mathrm{VS} / \mathrm{kg}$ culture/day and HRT of 28 days), where the amount of replaced materials was increased to $2.0 \mathrm{~g}$ TS of substrate, and the cultivation period was approximately 3.0 days (Fig. 1a). Methane production was stable until day 83, which corresponded to 1.3-fold HRT under condition 2 and 2.3-fold HRT under conditions 1 and 2 cumulatively (Fig. 1b). However, VFAs began to accumulate from day 86 (1.4-fold HRT under condition 2 and 2.4-fold HRT under conditions 1 and 2, cumulatively) with decreasing methane production. The salinity and VS content were nearly constant from day 86, suggesting that the accumulation of VFAs began from the initial steady-state phase under condition 2. The methane yield under condition 2 was $335 \pm 3 \mathrm{~mL} \mathrm{CH}_{4} / \mathrm{g} \mathrm{VS}$, which was $93.5 \%$ of that under condition 1, owing to incomplete methanization of VFAs. This result indicated that the yield decreased under condition 2, and that methane production became unstable during the transition from the non-steady state to the steady state.

To recover the methane yield, OLR was decreased to $1.7 \mathrm{~g} \mathrm{VS}$ substrate/kg culture/day by lengthening the HRT to 46 days (condition 3), where the amount of replaced materials was the same as under condition 2 (2.0 $\mathrm{g}$ TS of substrate with appropriate amount of anaerobic water) and the cultivation period was approximately 4.6 days (Fig. 1a). The methane yield was high at the beginning of condition 3 because 
the VFAs that accumulated during cultivation under condition 2 were converted to methane (Fig. 1b). Methane production, VS content, and salinity were stable throughout the 2.1-fold-HRT period of condition 3, which corresponded to 3.5- to 5.6-fold HRT under all the conditions, indicating that the cultivation under condition 3 was stably conducted under steady state. The methane yield was $346 \pm 5 \mathrm{~mL} \mathrm{CH}_{4} / \mathrm{g}$ VS, which was higher than that under condition $2\left(335 \pm 3 \mathrm{~mL} \mathrm{CH}_{4} / \mathrm{g} \mathrm{VS}\right)$, indicating that the methane yield was indeed recovered.

These results indicated that for steady-state, semi-continuous methane production from materials equivalent to raw brown algae, the OLR was limited to between $1.7 \mathrm{~g}$ VS/kg culture/day under condition 3 and $2.9 \mathrm{~g}$ VS substrate/kg culture/day under condition 2 .

\subsubsection{Estimation of the cause of unstable methane production}

The accumulation of VFAs during cultivation under condition 2 led to unstable methane production (Fig. 1a and 1b). Therefore, we investigated the cause of VFA accumulation.

In general, decreased $\mathrm{pH}$ inhibits methanogens utilizing acetate, resulting in accumulation of acetate. However, the $\mathrm{pH}$ did not decrease during the entire experiment (Fig. 1c) and thus, could not have affected methane production.

The salinity decreased throughout cultivation under conditions 1 and 2 from 3.8 to $1.9 \% \mathrm{NaCl}$ equivalent (Fig. 1c). The reduction in the salinity resulted from intermittent replacement of culture, because the starter culture contained salts equivalent to approximately $4 \% \mathrm{NaCl}$ as described in Section 2.3. Moreover, ash content of the materials added was approximately $2 \mathrm{wt} \%$, which was calculated from $10 \mathrm{wt} \% \mathrm{TS}$ 
content of the materials and approximately $20 \mathrm{wt} \%$ VS content of TS. The significant decrease in salinity might have affected methanogenic activity to cause unstable semi-continuous methane production. Thus, the effect of salinity on methanogenic activity was investigated. Acetoclastic methanogenic activity was measured at various salinity levels (Table 1) because acetoclastic methanogenesis is a rate-limiting step (Ito et al., 2012; Miura et al., 2014; Noike et al., 1985; Ras et al., 2011), causing accumulation of acetate as the major intermediate product (Fig. 1b). A culture removed at the first round of cultivation under condition 3 was used to measure acetate consumption rates. As shown in Table 1 , the acetate consumption rate at $2 \% \mathrm{NaCl}$ equivalent was higher than that at $4 \% \mathrm{NaCl}$ equivalent. This result indicated that acetoclastic methanogenesis was not inhibited by the decreased salinity. Thus, methane production was not affected by the significantly decreased salinity in the semi-continuous operation.

VS content of the culture significantly decreased from 3.4 to $1.9 \mathrm{wt} \%$ throughout cultivation under conditions 1 and 2 (Fig. 1c). Reduced VS resulted from washout of VS by the semi-continuous operation. Moreover, VS content reflects microbial content. Thus, the reduction in VS probably affected methane production. Indeed, the methane production rate decreased from $1386 \pm 61 \mathrm{~mL} \mathrm{CH}_{4} / \mathrm{kg}$ culture/day during initial batch cultivation to $745 \pm 37 \mathrm{~mL} \mathrm{CH}_{4} / \mathrm{kg}$ culture/day from day 177 to day 181 under condition 3. Furthermore, specific methane production rates $\left(\mathrm{mL} \mathrm{CH}_{4} / \mathrm{g}\right.$ VS culture/day) were almost the same in both conditions. These results suggested that the semi-continuous operation decreased microbial content, leading to decreased methane production with accumulation of mainly acetate from day 86 under condition 2, even though acetoclastic 
methanogenesis, which is a rate-limiting step during methane production, was enhanced by the decreased salinity.

\subsection{Microbial community}

\subsubsection{Bacterial community}

The numbers of bacterial chimera-filtered operational taxonomic units (OTUs) and reads in the chimera-filtered OTUs obtained for each of the cultivation conditions were as follows: 452 OTUs and 22,610 reads for the starter culture, 286 OTUs and 10,729 reads for culture under condition 1, 282 OTUs and 15,629 reads for culture under condition 2, and 216 OTUs and 18,069 reads for culture under condition 3 . The results indicated that the number of OTUs decreased in continuous cultivation compared to the number of OTUs in starter culture.

The abundance at phylum and family levels for the major bacterial taxa with a relative abundance $\geq 1 \%$ is shown in Fig. 2. Analysis of the bacterial phyla suggested that Fusobacteria was the predominant phylum throughout all culture conditions, although the abundance decreased in semi-continuous cultivation compared to the starter culture, and the Bacteroidetes gradually increased throughout the conditions to become another dominant phylum (Fig. 2a). The family Fusobacteriaceae was the only component of the phylum Fusobacteria, and the increase in the phylum Bacteroidetes could be mainly attributed to bacteria of the order Bacteroidales (Fig. 2b). The members of the family Fusobacteriaceae of the phylum Fusobacteria can ferment carbohydrate into acids (Nelson et al., 2013). The Bacteroidetes have the capacity to degrade polysaccharides (Hanreich et al., 2013). Thus, these predominant bacterial phyla may be involved in the conversion of carbohydrates. 
Bacteria involved in acetogenesis were undetected. In the acetogenesis step, propionate and butyrate are oxidized to acetate by depletion of hydrogen produced from the VFAs by hydrogenotrophic methanogens. Known propionate oxidizers are Syntrophobacter spp., Smithella propionica, Pelotomaculum spp., and Desulfotomaculum spp., and known butyrate oxidizers are S. propionica, Desulfotomaculum spp., Syntrophus aciditrophicus, Algorimarina butyrica, Syntrophomonas spp., Thermosyntropha spp., and Syntrophothermus lipocalidus (Stams et al., 2012). All species were undetected in this study. However, the genus Pelotomaculum possibly involved in propionate oxidation and the genus Syntrophomonas possibly involved in butyrate oxidation were detected with abundances of less than $0.1 \%$ and $0.2 \%$, respectively, throughout all culture conditions. This is in accordance with a previous study in which these species were detected in methanogenic digesters at less than $2 \%$ (Stams et al., 2012).

\subsubsection{Archaeal community}

The numbers of archaeal chimera-filtered OTUs and reads in the chimera-filtered OTUs obtained for each of the cultivation conditions were as follows: 48 OTUs and 19,678 reads for the starter culture, 37 OTUs and 11,723 reads for culture under condition 1, 42 OTUs and 13,225 reads for culture under condition 2, and 32 OTUs and 15,764 reads for culture under condition 3 . These results indicated that the number of OTUs did not significantly change under the different culture conditions.

The relative abundance at the genus level for the major archaeal taxa with a relative abundance $\geq 1 \%$ is shown in Fig. 3. Analysis of the archaeal taxa suggested that Methanosaeta, the acetoclastic methanogen, was predominant in the starter culture and 
under condition 1 , indicating that methane was directly produced from acetate, the major intermediate product, by Methanosaeta. However, subsequent operation at the highest OLR under condition 2 changed the archaeal community; the abundance of Methanosaeta decreased and Methanoculleus, a hydrogenotrophic methanogen, became predominant. An increase in hydrogenotrophic methanogens under increased OLR has been observed in other studies (Blume et al., 2010; Lerm et al., 2012; Razaviarani and Buchanan, 2014). Further operation under condition 3 did not recover the relative abundance of Methanosaeta despite recovery of methane production, but further enhanced the predominance of Methanoculleus. Methanosarcina, another acetoclastic methanogen, was a minor genus throughout all the conditions. These results implied that the methanogenic pathway partially shifted from direct conversion of acetate to methane by acetoclastic methanogens to an indirect pathway involved in hydrogenotrophic methanogens.

In general, hydrogenotrophic methanogens can grow syntrophically with acetate oxidizers (Hattori, 2008). Oxidation of acetate to carbon dioxide and hydrogen is thermodynamically unfavorable, but becomes feasible by depleting hydrogen. Hydrogenotrophic methanogens enable acetate oxidation by acetate oxidizers by converting hydrogen to methane. Therefore, the predominance of the hydrogenotrophic methanogens indicated that methane might have been produced via acetate oxidation and hydrogenotrophic methanogenesis during cultivation under conditions 2 and 3 in addition to via acetoclastic methanogenesis. This hypothesis is supported by a study that showed that possible acetate oxidizers and hydrogenotrophic methanogens increased with increasing OLR during anaerobic digestion of food wastewater (Jang et al., 2014). 
The bacterial phyla Firmicutes, Spirochaetes, Synergistetes, and Thermotogae, which are involved in the oxidation of acetate under thermophilic and/or mesophilic conditions, increased under the highest OLR in condition 2 (Fig. 2a). However, no known acetate oxidizers were detected in the culture under the mesophilic condition at high salinity levels.

Bacteria within the family Lachnospiraceae were mainly responsible for the increase in the phylum Firmicutes under the mesophilic condition (Fig. 2b). The phylum Firmicutes contained known acetate oxidizers: a strain from Clostridium ultunense (Schnürer et al., 1996) belonging to the family Clostridiaceae, a strain from Thermacetogenium phaeum (Hattori et al., 2000) and strains from Tepidanaerobacter acetatoxydans (Westerholm et al., 2011) of the family Thermoanaerobacteraceae, and a strain from Syntrophaceticus schinkii (Westerholm et al., 2010) of the family Thermoanaerobacterales (Family III). Among these strains, C. ultunense is the only known acetate oxidizer under mesophilic conditions among these strains. Thus, bacteria of the family Lachnospiraceae may not be the acetate oxidizers in this study.

Bacteria classified into the families Sphaerochaetaceae and Spirochaetaceae were responsible for the increase in the phylum Spirochaetes (Fig. 2b). The Spirochaetes are known to include acetate oxidizers, both in mesophilic and thermophilic conditions, but are not further classified (Lee et al., 2015). Thus, bacteria of the phylum Spirochaetes might be unknown acetate oxidizers in this study.

Bacteria of the family Aminiphilaceae were mainly responsible for the increase in Synergistetes, and the family Thermotogaceae for the increase in Thermotoga (Fig. 2b). The phylum Synergistetes contains known acetate oxidizers, which form the Synergistes group 4 (Ito et al., 2011) of the family Synergistaceae. The phylum Thermotoga 
contains a known acetate oxidizer, Thermotoga lettingae (Balk et al., 2002), which belongs to the family Thermotogaceae. Those strains were obtained from non-mesophilic conditions (Balk et al., 2002; Ito et al., 2011). However, resolution to the genus level showed that bacteria of the genus Kosmotoga were mainly responsible for the increase in the family Thermotogaceae. Therefore, bacteria of the families Aminiphilaceae and Thermotogaceae may not be the acetate oxidizers in this study.

The results indicated that unknown acetate oxidizers of the Spirochaetes, which increased under the highest OLR in condition 2 leading to unstable methane production, might have caused a partial shift of methanogenic pathway from acetoclastic methanogenesis to hydrogenotrophic methanogenesis via acetate oxidation.

\subsection{Comparison of the ability to produce methane from brown algae}

The performance of methane production under the conditions described in this study was compared to that in other studies that also used semi-continuous methane production from brown algae under mesophilic conditions (Table 2). In this study, methane was produced from materials equivalent to raw brown algae by sediment microbes without nutrient supply at 3 consecutive different OLRs as follows: stable methane production without accumulation of VFA at OLR of $2.0 \mathrm{~g}$ VS/ $\mathrm{kg}$ culture/day under non-steady state condition, unstable methane production with accumulation of VFAs at subsequent OLR of $2.9 \mathrm{~g} \mathrm{VS} / \mathrm{kg}$ culture/day during the transition from non-steady to steady state, and stable methane production without accumulation of VFAs at subsequent OLR of $1.7 \mathrm{~g}$ VS/kg culture/day under steady state.

In previous studies, raw algal feedstocks were generally diluted with water (Hanssen et al., 1987; Hinks et al., 2013) (Table 2). The OLR for stable methane production under 
undiluted conditions in this study (1.7-2.0 $\mathrm{g} \mathrm{VS} / \mathrm{kg}$ culture/day) was comparable to or higher than that under diluted conditions with or without nutrient supply in the other studies.

Jard et al. (2012) performed methane production under undiluted conditions (Table 2). However, the methane yield in the study rapidly decreased during the non-steady state, between 1.3- and 1.9-fold HRT, and the highest OLR was $2.2 \mathrm{~g} \mathrm{VS/L} \mathrm{culture/day,}$ despite the nutrient supply (Jard et al., 2012). In contrast, in our study, the methane yield was stable during the non-steady state, but gradually decreased from the beginning of the steady state during the highest OLR of $2.9 \mathrm{~g} \mathrm{VS} / \mathrm{kg}$ culture/day. These findings indicate that the performance of methane production from raw brown algae without dilution in our study was higher than that in the previous study.

The achievable methane production rate was estimated by multiplying the OLR by the methane yield (Table 2). Comparison of the methane production rates showed that the ability to produce methane from brown algae in the current study was the highest among the studies, which may be attributable to differences in the starter culture used. In this study, acclimated culture derived from marine sediment was used. In contrast, in the previous studies, a culture from non-marine origin was used. The non-marine microbes are likely less resistant to the salts derived from the brown algae than those of marine origin.

\section{Conclusions}

Methane was produced semi-continuously from brown algae under undiluted conditions by acclimated marine sediment-derived culture beyond steady state. Microbial community analysis implied that the methanogenic pathway remained 
partially shifted from acetoclastic methanogenesis to acetate oxidation from increased OLR. The ability of the acclimated culture to produce methane semi-continuously was higher than that observed in previous studies using cultures of non-marine origin. The OLR is limited by the HRT in the case of marine macroalgae because of their high water content. Therefore, future studies should aim to increase the OLR by shortening the HRT.

\section{Acknowledgments}

This work was supported by the Core Research of Evolutional Science and Technology program (CREST) from the Japan Science and Technology Agency (JST).

\section{References}

1 Balk, M., Weijma, J., Stams, A.J.M., 2002. Thermotoga lettingae sp. nov., a novel thermophilic, methanol-degrading bacterium isolated from a thermophilic anaerobic reactor. Int. J. Syst. Evol. Microbiol. 52, 1361-1368.

2 Blume, F., Bergmann, I., Nettmann, E., Schelle, H., Rehde, G., Mundt, K., Klocke, M., 2010. Methanogenic population dynamics during semi-continuous biogas fermentation and acidification by overloading. J. Appl. Microbiol. 109, $441-450$.

3 Briand, X., Morand, P., 1997. Anaerobic digestion of Ulva sp. 1. Relationship between Ulva composition and methanisation. J. Appl. Phycol. 9, 511-524.

4 Chynoweth, D.P., Owens, J.M., Legrand, R., 2001. Renewable methane from anaerobic digestion of biomass. Renew. Energy 22, 1-8. 
5 Feijoo, G., Soto, M., Méndez, R., Lema, J.M., 1995. Sodium inhibition in the anaerobic digestion process: antagonism and adaptation phenomena. Enzyme and Microbial Technology 17, 180-188.

6 Hanreich, A., Schimpf, U., Zakrzewski, M., Schlüter, A., Benndorf, D., Heyer, R., Rapp, E., Pühler, A., Reichl, U., Klocke, M., 2013. Metagenome and metaproteome analyses of microbial communities in mesophilic biogas-producing anaerobic batch fermentations indicate concerted plant carbohydrate degradation. Syst. Appl. Microbiol. 36, 330-338.

7 Hanssen, J.F., Indergaard, M., Østgaard, K., Bævre, O.A., Pedersen, T.A., Jensen, A., 1987. Anaerobic digestion of Laminaria spp. and Ascophyllum nodosum and application of end products. Biomass 14, 1-13.

8 Hattori, S., 2008. Syntrophic acetate-oxidizing microbes in methanogenic environments. Microbes Environ. 23, 118-127.

9 Hattori, S., Kamagata, Y., Hanada, S., Shoun, H., 2000. Thermacetogenium phaeum gen. nov., sp. nov., a strictly anaerobic, thermophilic, syntrophic acetate-oxidizing bacterium. Int. J. Syst. Evol. Microbiol. 50, 1601-1609.

10 Hinks, J., Edwards, S., Sallis, P.J., Caldwell, G.S., 2013. The steady state anaerobic digestion of Laminaria hyperborea - Effect of hydraulic residence on biogas production and bacterial community composition. Bioresour. Technol. $143,221-230$.

11 Ito, T., Yoshiguchi, K., Ariesyady, H.D., Okabe, S., 2012. Identification and quantification of key microbial trophic groups of methanogenic glucose degradation in an anaerobic digester sludge. Bioresour. Technol. 123, 599-607. 
12 Ito, T., Yoshiguchi, K., Ariesyady, H.D., Okabe, S., 2011. Identification of a novel acetate-utilizing bacterium belonging to Synergistes group 4 in anaerobic digester sludge. ISME J. 5, 1844-1856.

13 Jang, H.M., Kim, J.H., Ha, J.H., Park, J.M., 2014. Bacterial and methanogenic archaeal communities during the single-stage anaerobic digestion of high-strength food wastewater. Bioresour. Technol. 165, 174-182.

14 Jard, G., Jackowiak, D., Carrère, H., Delgenes, J.P., Torrijos, M., Steyer, J.P., Dumas, C., 2012. Batch and semi-continuous anaerobic digestion of Palmaria palmata: comparison with Saccharina latissima and inhibition studies. Chem. Eng. J. 209, 513-519.

15 Lee, S.-H., Park, J.-H., Kim, S.-H., Yu, B.J., Yoon, J.-J., Park, H.-D., 2015. Evidence of syntrophic acetate oxidation by Spirochaetes during anaerobic methane production. Bioresour. Technol. 190, 543-549.

16 Lerm, S., Kleyböcker, A., Miethling-Graff, R., Alawi, M., Kasina, M., Liebrich, M., Würdemann, H., 2012. Archaeal community composition affects the function of anaerobic co-digesters in response to organic overload. Waste Manag. 32, 389-399.

17 Liu, Y.T., Boone, D.R., 1991. Effects of salinity on methanogenic decomposition. Bioresour. Technol. 35, 271-273.

18 Miura, T., Kita, A., Okamura, Y., Aki, T., Matsumura, Y., Tajima, T., Kato, J., Nakashimada, Y., 2014. Evaluation of marine sediments as microbial sources for methane production from brown algae under high salinity. Bioresour. Technol. 169, 362-366. 
19 Miura, T., Kita, A., Okamura, Y., Aki, T., Matsumura, Y., Tajima, T., Kato, J., Nakashimada, Y., 2015. Improved methane production from brown algae under high salinity by fed-batch acclimation. Bioresour. Technol. 187, 275-281.

20 Montingelli, M.E., Tedesco, S., Olabi, A.G., 2015. Biogas production from algal biomass: a review. Renew. Sust. Energy Rev. 43, 961-972.

21 Mottet, A., Habouzit, F., Steyer, J.P., 2014. Anaerobic digestion of marine microalgae in different salinity levels. Bioresour. Technol. 158, 300-306.

22 Nelson, T.M., Rogers, T.L., Brown, M.V., 2013. The gut bacterial community of mammals from marine and terrestrial habitats. PLoS One 8, e83655.

23 Noike, T., Endo, G., Chang, J.-E., Yaguchi, J., Matsumoto, J., 1985. Characteristics of carbohydrate degradation and the rate-limiting step in anaerobic digestion. Biotechnol. Bioeng. 27, 1482-1489.

24 Ras, M., Lardon, L., Bruno, S., Bernet, N., Steyer, J.-P., 2011. Experimental study on a coupled process of production and anaerobic digestion of Chlorella vulgaris. Bioresour. Technol. 102, 200-206.

25 Razaviarani, V., Buchanan, I.D., 2014. Reactor performance and microbial community dynamics during anaerobic co-digestion of municipal wastewater sludge with restaurant grease waste at steady state and overloading stages. Bioresour. Technol. 172, 232-240.

26 Rinzema, A., van Lier, J., Lettinga, G., 1988. Sodium inhibition of acetoclastic methanogens in granular sludge from a UASB reactor. Enzyme and Microbial Technology 10, 24-32. 
27 Roesijadi, G., Jones, S.B., Snowden-Swan, L.J., Zhu, Y., 2010. Macroalgae as a biomass feedstock: a preliminary analysis. Pacific Northwest National Laboratory, Richland, WA.

28 Schnürer, A., Schink, B., Svensson, B.H., 1996. Clostridium ultunense sp. nov., a mesophilic bacterium oxidizing acetate in syntrophic association with a hydrogenotrophic methanogenic bacterium. Int. J. Syst. Bacteriol. 46, $1145-1152$.

29 Stams, A.J.M., Sousa, D.Z., Kleerebezem, R., Plugge, C.M., 2012. Role of syntrophic microbial communities in high-rate methanogenic bioreactors. Water Sci. Technol. 352-362.

30 Westerholm, M., Roos, S., Schnürer, A., 2010. Syntrophaceticus schinkii gen. nov., sp. nov., an anaerobic, syntrophic acetate-oxidizing bacterium isolated from a mesophilic anaerobic filter. FEMS Microbiol. Lett. 309, 100-104.

31 Westerholm, M., Roos, S., Schnürer, A., 2011. Tepidanaerobacter acetatoxydans sp. nov., an anaerobic, syntrophic acetate-oxidizing bacterium isolated from two ammonium-enriched mesophilic methanogenic processes. Syst. Appl. Microbiol. 34, 260-266. 


\section{Figure captions}

Fig. 1. Semi-continuous methane production from brown algae under 3 different undiluted conditions. (a) OLR and HRT. (b) Methane production and VFA accumulation. (c) $\mathrm{pH}$, salinity, and VS.

Fig. 2. Relative abundance of bacterial taxa at the phylum (a) and family (b) levels. Taxa with an abundance of $<1 \%$ were grouped as minor taxa.

Fig. 3. Relative abundance of archaeal taxa at the genus level. Taxa with an abundance of $<1 \%$ were grouped as minor taxa. 

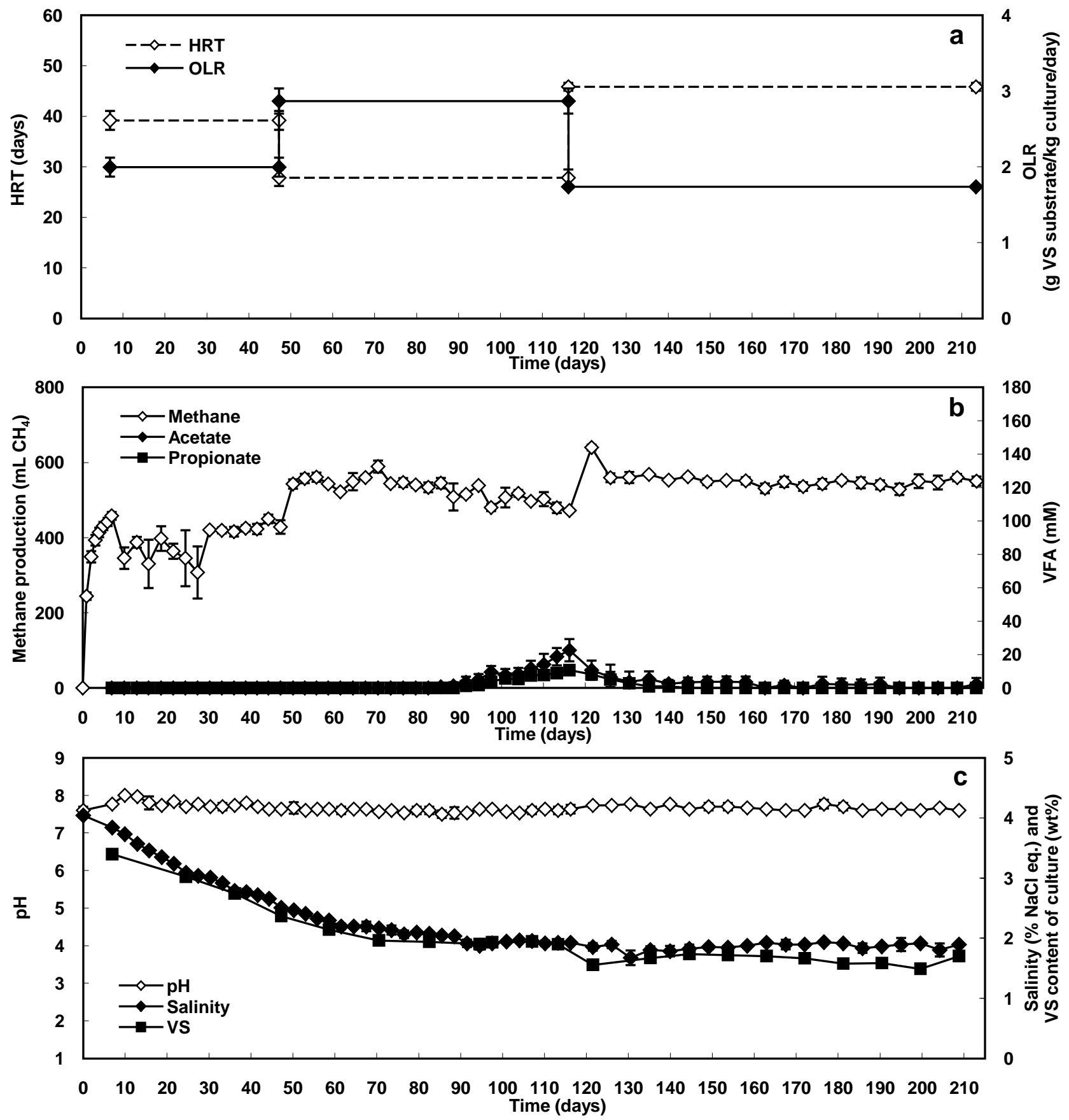




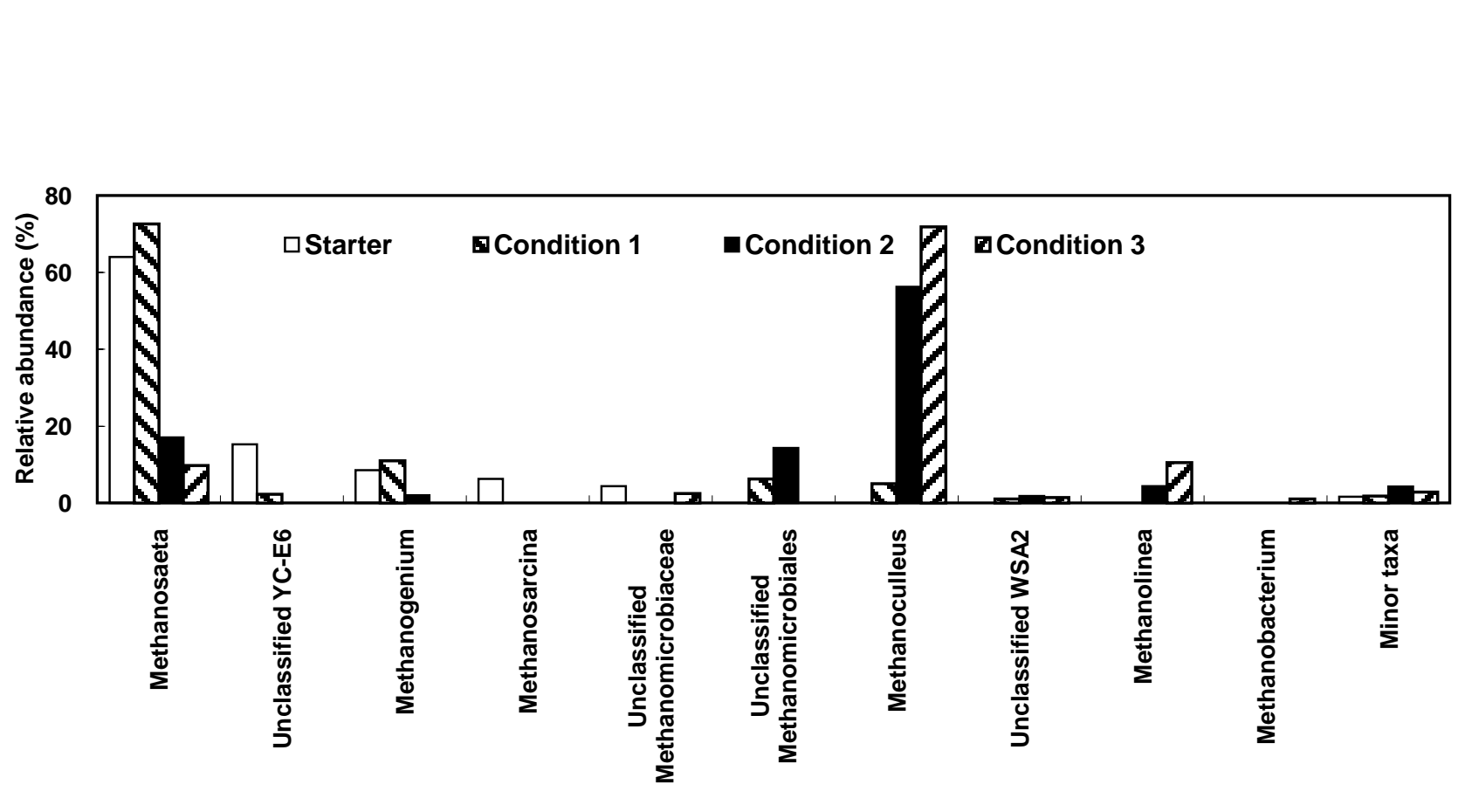

Figure3

Figure3

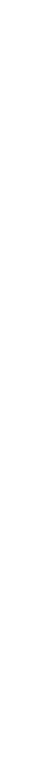

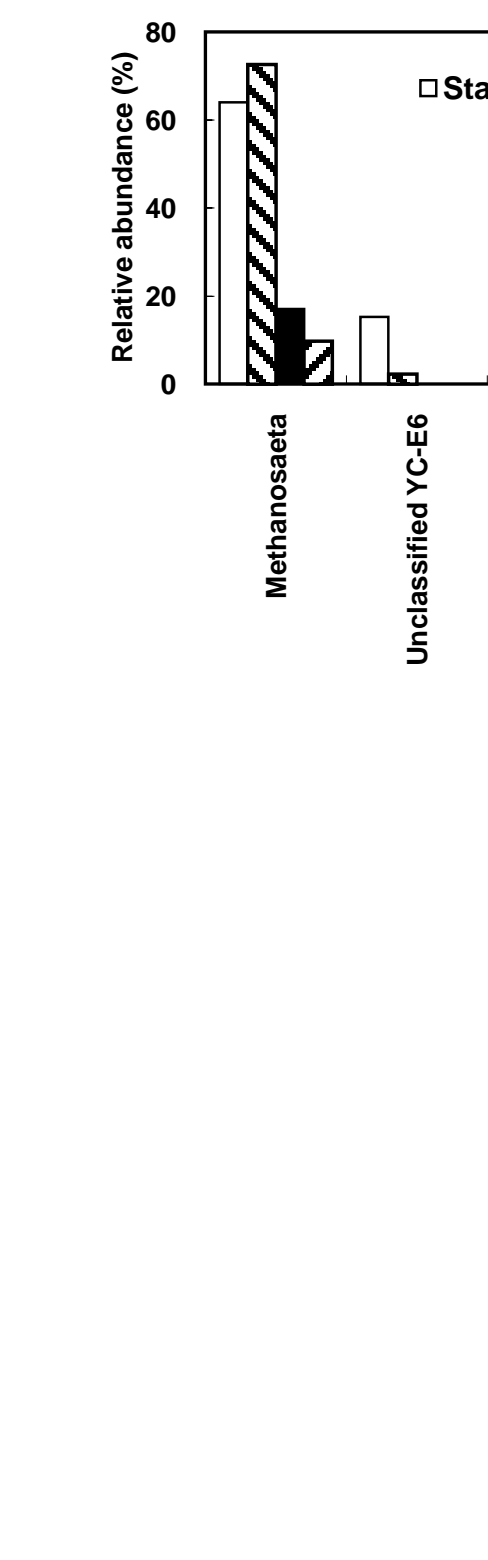




\section{Table 1}

Acetate consumption rate at various salinity levels.

\begin{tabular}{lll}
\hline $\mathrm{NaCl}(\%)$ & $\begin{array}{l}\text { Acetate consumption rate } \\
(\mathrm{mmol} / \mathrm{L} / \text { day })\end{array}$ & Relative activity $(\%)$ \\
\hline 4 & $7.5 \pm 0.9$ & $100 \pm 12$ \\
3 & $7.9 \pm 0.3$ & $105 \pm 4$ \\
2 & $9.3 \pm 0.6$ & $124 \pm 8$ \\
1 & $13.6 \pm 0.6$ & $181 \pm 8$ \\
\hline
\end{tabular}




\section{Table 2}

Comparison of methane production from various species of brown algae.

\begin{tabular}{|c|c|c|c|c|c|c|c|}
\hline Substrate & $\begin{array}{l}\text { OLR (g } \\
\text { VS/L/day) }\end{array}$ & $\begin{array}{l}\text { Nutrient } \\
\text { supply }\end{array}$ & $\begin{array}{l}\text { Methane } \\
\text { yield (mL/g } \\
\text { VS) }\end{array}$ & HRT (days) & $\begin{array}{l}\text { State (-fold } \\
\text { HRT) }\end{array}$ & $\begin{array}{l}\text { Methane } \\
\text { production rate } \\
(\mathrm{mL} / \mathrm{L} / \mathrm{day})^{\mathrm{b}}\end{array}$ & Reference \\
\hline \multicolumn{8}{|l|}{ Diluted conditions } \\
\hline Laminaria hyperborea & 1.65 & - & 280 & 24 & $\mathrm{ND}^{\mathrm{c}}$ & 462 & $\begin{array}{l}\text { (Hanssen et } \\
\text { al., 1987) }\end{array}$ \\
\hline Laminaria saccharina & 1.65 & - & 230 & 24 & ND & 380 & $\begin{array}{l}\text { (Hanssen et } \\
\text { al., 1987) }\end{array}$ \\
\hline Ascophyllum nodosum & 1.75 & - & 110 & 24 & ND & 193 & $\begin{array}{l}\text { (Hanssen et } \\
\text { al., 1987) }\end{array}$ \\
\hline Laminaria hyperborea & 1.00 & + & 230 & 20 & Steady $(0-3)$ & 230 & $\begin{array}{l}\text { (Hinks et } \\
\text { al., 2013) }\end{array}$ \\
\hline \multicolumn{8}{|l|}{ Undiluted conditions } \\
\hline Saccharina latissima & 1.73 & + & 238 & 38 & $\begin{array}{l}\text { Non-steady } \\
(0-1.3)\end{array}$ & 412 & $\begin{array}{l}\text { (Jard et al., } \\
\text { 2012) }\end{array}$ \\
\hline & 2.17 & + & 137 & 33 & $\begin{array}{l}\text { Non-steady } \\
(1.3-1.9)\end{array}$ & 297 & $\begin{array}{l}\text { (Jard et al., } \\
\text { 2012) }\end{array}$ \\
\hline
\end{tabular}




\begin{tabular}{|c|c|c|c|c|c|c|c|}
\hline \multirow[t]{3}{*}{ Saccharina japonica } & 2.00 & - & 358 & 39 & $\begin{array}{l}\text { Non-steady } \\
(0-1.0)\end{array}$ & 695 & This study \\
\hline & 2.87 & - & 335 & 28 & $\begin{array}{l}\text { Steady } \\
(1.0-3.5)^{\mathrm{d}}\end{array}$ & 961 & This study \\
\hline & 1.74 & - & 346 & 46 & $\begin{array}{l}\text { Steady } \\
(3.5-5.6)\end{array}$ & 602 & This study \\
\hline
\end{tabular}

Methane was produced under mesophilic conditions.

${ }^{\mathrm{a}, \mathrm{b}}$ The unit of OLR and methane production rate in this study were $\mathrm{g} \mathrm{VS} / \mathrm{kg} / \mathrm{day}$ and $\mathrm{mL} / \mathrm{kg} / \mathrm{day}$, respectively.

${ }^{\mathrm{b}}$ The rate was calculated by multiplying the OLR by the methane yield.

${ }^{\mathrm{c}} \mathrm{ND}$, not described.

${ }^{\mathrm{d}}$ VFAs began to accumulate from 2.4-fold HRT. 